\title{
Multiplexing structured illumination in spatial light modulators to achieve super-resolution
}

\section{Imágenes super-resueltas obtenidas mediante iluminación estructurada generada con moduladores espaciales de luz}

\author{
Anwar Hussain(1,3), José L. Martínez ${ }^{(2, S)}$, A. Lizana $\left(3,{ }^{(3)}\right.$, Juan Campos ${ }^{(3, S)}$ \\ 1. Department of Physics and Applied Mathematics, Pakistan Institute of Engineering and Applied Sciences. Nilore \\ 45650, Islamabad, Pakistan. \\ 2. Departamento de Ciencia de Materiales, Óptica y Tecnología Electrónica, Universidad Miguel Hernández, 03202 \\ Elche, Alicante, Spain. \\ 3. Departameno de Física. Universitat Autònoma de Barcelona, 08193, Bellaterra, Barcelona, Spain. \\ (*) Email: angel.lizana@uab.es S: miembro de SEDOPTICA / SEDOPTICA member \\ Recibido / Received: 23/11/2012. Revisado / Revised: 14/03/2013. Aceptado / Accepted: 16/03/2013. \\ DOI: http://dx.doi.org/10.7149/OPA.46.2.165
}

\begin{abstract}
:
We present some numerical results corresponding to a recently proposed structured illumination based set-up able to achieve resolution beyond the diffraction limit in $4 f$ optical setup. The technique is based on the use of a liquid crystal display to multiplex the different linear phases which produce all the tilted plane waves at the same time. At the SLM plane constant phases are assigned to each tilted beam. The object is illuminated several times with simultaneous illumination (i.e., simultaneous tilted beams), every time carrying different constant phases. A total of $n(n-1)+1$ interferograms are recorded at the CCD plane, corresponding to the same number of constant phases sets. The proper selection of phases helps to separate the correlated terms. In a post processing procedure, each hologram is transformed to the spectrum domain and spatially shifted to its proper position. The combination of these shifted segments of the object spectrum gives the synthesized spectrum. To obtain super resolved image inverse Fourier transform is taken of the synthesized spectrum.
\end{abstract}

Key words: Spatial Light Modulators, Super-Resolution, Multiplexed Illumination.

\section{RESUMEN:}

En este trabajo se presentan resultados numéricos correspondientes a un nuevo método para la obtención de imágenes super-resueltas que se basa en la iluminación estructurada del objeto a estudiar. En particular, la iluminación estructurada consiste en diferentes ondas planas con distintas inclinaciones que iluminan el objeto simultáneamente. Para generar dicha iluminación, se utiliza una pantalla de cristal líquido donde se multiplexan diferentes fases lineales, que originan las distintas ondas planas simultáneamente. Para recuperar apropiadamente la información extra de las frecuencias altas del objeto, que está codificada en los diferentes haces inclinados, se ilumina el objeto diversas veces con la iluminación estructurada, pero añadiendo diferentes fases constantes a los distintos haces. Los correspondientes interferogramas se capturan mediante el uso de una cámara CCD. La selección adecuada de los valores para las fases constantes añadidas, permite separar los diferentes términos correlacionados. Finalmente, en un post-procesado, se realiza la Transformada de Fourier de cada holograma obtenido, y se desplaza cada fragmento del dominio de frecuencias del objeto a su posición correcta. Aplicando la transformada de Fourier inversa al espectro sintetizado, obtenemos una imagen super-resuelta del objeto.

Palabras clave: Modulador Espacial de Luz, Super-Resolución, Iluminación Multiplexada. 


\section{REFERENCIAS Y ENLACES / REFERENCES AND LINKS}

[1]. P. Ferraro, A. Wax, Z. Zalevsky, D. Mendlovic, Edts., Coherent Light Microscopy: Imaging and Quantitative Phase Analysis, Springer, Heidelberg, Germany(2011). DOI

[2]. M. Paturzo, F. Merola, S. Grilli, S. De Nicola, A. Finizio, P. Ferraro, "Super-resolution in digital holography by a two dimensional dynamic phase grating", Opt. Express 16,17107-17118 (2008). DOI

[3]. A. Mudassar, A. R. Harvey, A. H. Greenaway, J. D. C. Jones, "Resolution beyond classical limits with spatial frequency heterodyning", Chin. Opt. Lett. 4, 148-151 (2006).

[4]. A. A. Mudassar, H. Anwar, "Super-resolution of active spatial frequency heterodyning using holographic approach", Appl. Opt. 49, 3434-3441 (2010). DOI

[5]. M. G. L. Gustafsson, "Surpassing the lateral resolution limit by a factor of two using structured illumination microscopy", J. Microsc. 198, 82-87 (2000). DOI

[6]. M. Ueda, T. Sato, "Superresolution by holography", J. Opt. Soc. Am. 61, 418-419 (1971). DOI

[7]. M. Ueda, T. Sato, M. Kondo, "Superresolution by multiple superposition of image holograms having different carrier frequencies", J. Mod. Opt. 20, 403-410 (1973).

[8]. A. Hussain, A. A. Mudassar, "Holography based super resolution", Opt. Commun. 285, 2303-2310 (2012). DOI

[9]. A.-H. Phan, J.-H. Park, N. Kim "Super-resolution digital holographic microscopy for three dimensional sample using multipoint light source illumination", Jap. J. Appl. Phys. 50, 092503 (2011). DOI

[10].V. Mico, Z. Zalevsky, P. García-Martínez, J. García, "Singe step superresolution by interferometric imaging", Opt. Express 12, 2589-2596 (2004). DOI

[11].A. Neumann, Y. Kuznetsova, S. R. J. Brueck, "Structured illumination for the extension of imaging interferometric microscopy", Opt. Express 16, 6785-6793 (2008). DOI

[12]. P. J. Valle, M. P. Cagigal "Analytic design of multiple-axis, diffractive multifocal lenses", Opt. Lett. 37, 1121-1123 (2012). DOI

[13]. A. Hussain, J. L. Martínez, A. Lizana, J. Campos, "Super resolution imaging achieved by using on-axis interferometry based on a spatial light modulator", Opt. Express 21, 9615-9623 (2013). D0I

[14]. C. Iemmi, J. Campos, J. C Escalera, O. López-Coronado, R. Gimeno, M. J. Yzuel, “Depth of focus increase by multiplexing programmable diffractive lenses", Opt. Express 14, 10207-10219 (2006). DOI

\section{Introduction}

The resolution of an optical imaging system can be defined as the smallest spatial separation distance between two features that still can be resolved in the imaging system. This resolution limit is depended upon the imaging wavelength and size of aperture and CCD sampling. The higher spatial frequencies of the object are blocked by the numerical aperture of the optical system. To obtain these high spatial frequencies of the object by breaking this classical limit is called superresolution [1]. Superresolution technique is applied in field where detailed information of the object is required, like medical imaging. Different techniques in literature have been reported to obtain high resolution images. Some of these techniques are based on grating [2], structure illumination [34], optical mask [5]. Holography is used to record the phase and amplitude of the object, firstly used for super resolution [6-7] in 1971.
The digital holography techniques are used to bring the higher spatial frequencies into the pass band using structure illumination [8-9]. The structure illumination is produced by different method like Vertical-cavity surface-emitting laser (VCSEL), grating or lens array. Usually in structured illumination imaging technique the object is illuminated from different angles sequentially, and interfered with reference beam to obtain interferogram $[5,10,11]$. In ref. [12], multiple focal point technique used to design amplitude and phases lenses.

Recently, we have proposed a new superresolution technique based on a spatial light modulator [13]. In the proposed technique, the object is illuminated from different defined directions and several interferograms are obtained by their mutual interference. Spatial liquid crystal modulator is used to create and multiplex these beams. Several images are captured by change of the relative phases. After 
proper post processing different parts of spectrum are shifted to their proper position to synthesize the spectrum, which inverse Fourier transform gives super resolved image. In this article we review this technique and present some additional numerical results.

\section{Optical set-up}

The used optical set-up to obtain high resolution image was proposed in Ref. [13] and it is shown in Fig. 1. An imaging $4 f$ optical system forms the image of the object on the CCD camera. At the Fourier plane an aperture is placed, blocking the high spatial frequencies of the object spectrum due to its limited size. This results in a low resolution image at the CCD plane. If the object is illuminated by a tilted plane wave, then, the Fourier spectrum of the object is shifted, allowing the pass of higher band frequencies of the object spectrum. The tilted plane wave is produced by the combination of the spatial light modulator (SLM) and the collimating lens. In fact, several divergent lenses with different shifts are encoded in the SLM. The back focal plane of the encoded lenses coincides with the focal plane of the collimating lens, producing a tilted plane wave whose tilt depends on the shift selected. In this way at the CCD plane images with different band pass can be obtained. By properly combining these images, a high resolution image can be produced.

We propose to use a multiplexed illumination with the 5 shifted lenses implemented at the same time. During this process the central part of object spectrum and also the other shifted spectrum segments are passing simultaneously through the imaging system. The random multiplexing method used here is explained in [14]. To retrieve all the correlation terms some constant phases are assigned to each multiplexed section of SLM plane. By using different combination of constant phases a total of seven interferograms are recorded for 1D (one dimensional case) and 21 interferograms for 2D (i.e. two dimensional case). From these recorded interferograms the correlations terms are retrieved. The recovered parts are shifted to their original position using the data of aperture

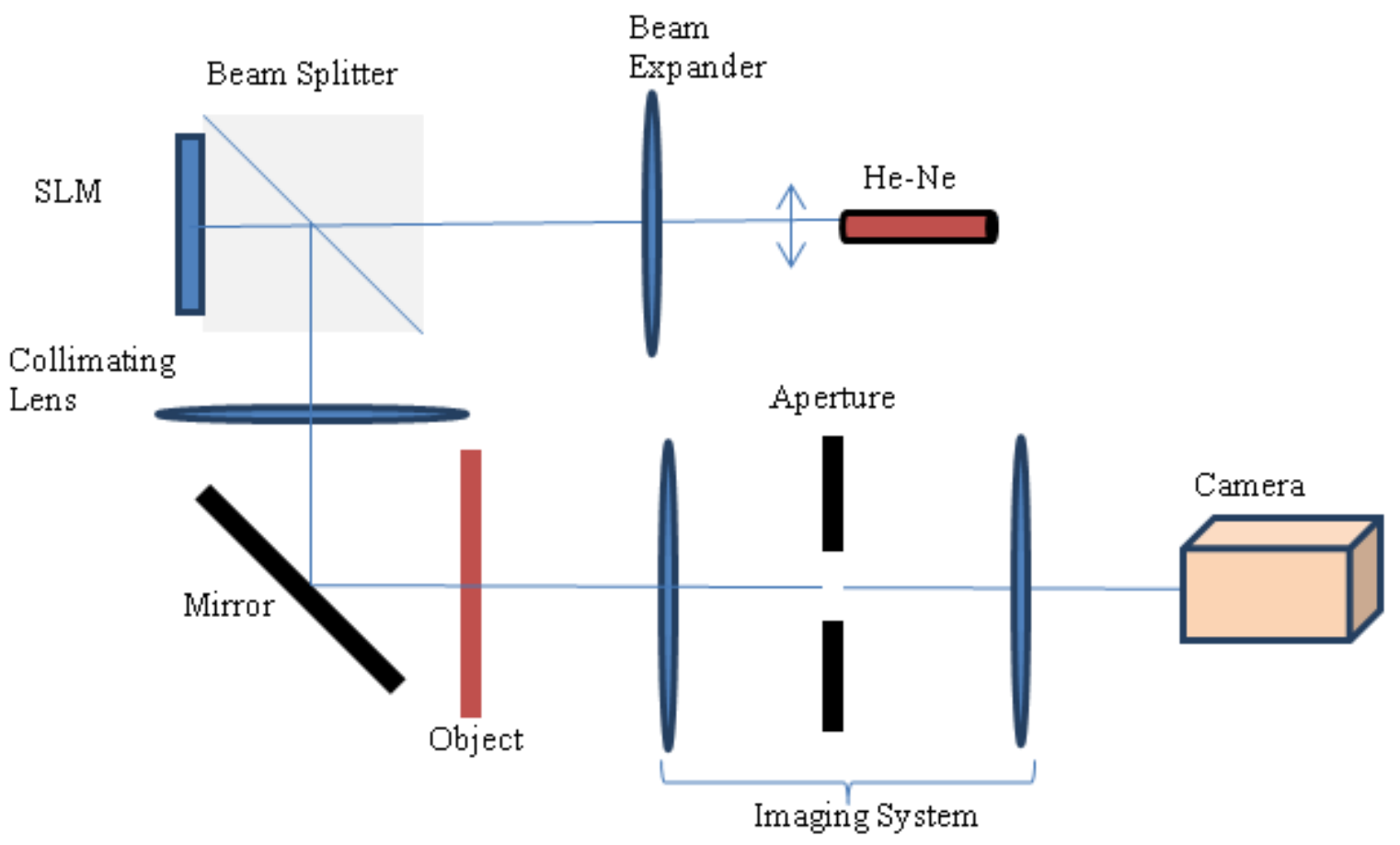

Fig. 1. Experimental set-up. 
size and SLM shift of tilted beams to reconstruct the synthesized spectrum, whose inverse Fourier transform gives super resolved image.

\section{Mathematical model}

Let us assume that the system in Fig. 1 is illuminated with plane waves without tilt. Then, the irradiance at the image plane will be:

$$
I(x, y)=|O(x, y) \otimes h(x, y)|^{2},
$$

where $O(x, y)$ and $h(x, y)$ are object transmission and the coherent point spread function (PSF) of the system respectively. Note that the sign $\otimes$ denotes convolution.

The Fourier transform of Eq. (1) with tilted beam illumination with constant phases $\delta_{0}$ is given as:

$$
\begin{aligned}
\tilde{I}_{0}(u, v)= & \left(e^{i \delta_{0}} \tilde{O}\left(u-u_{0}, v-v_{0}\right) H(u, v)\right) * \\
& *\left(e^{i \delta_{0}} \tilde{O}\left(u-u_{0}, v-v_{0}\right) H(u, v)\right),
\end{aligned}
$$

where the $\operatorname{sign} *$ denotes correlation. If the object is simultaneously illuminated with $n$ tilted beams having different constant phases, the corresponding Fourier transform is modified by:

$$
\begin{gathered}
\tilde{I}_{t}(u, v)=\left(\sum_{p=1}^{n} e^{i \delta_{p}} \tilde{O}\left(u-u_{p}, v-v_{p}\right) H(u, v)\right) *\left(\sum_{k=1}^{n} e^{i \delta_{k}} \tilde{O}\left(u-u_{k}, v-v_{k}\right) H(u, v)\right)= \\
=\sum_{p=1}^{n} \sum_{k=1}^{n} e^{i\left(\delta_{p}-\delta_{k}\right)}\left(\tilde{O}\left(u-u_{p}, v-v_{p}\right) H(u, v)\right) *\left(\tilde{O}\left(u-u_{k}, v-v_{k}\right) H(u, v)\right)= \\
=\sum_{p=1}^{n} \sum_{k=1}^{n} e^{i\left(\delta_{p}-\delta_{k}\right)} C_{p, k},
\end{gathered}
$$

where $p$ and $k$ denote the different phases assigned to the beams (which allow us to separate the correlated terms), and the coefficients $C_{p, k}$ accounts for the different correlations.

For the two-dimensional case, we have created a total of five beams $(n=5)$, two tilted beams leading to displacements at the $x$ direction, two tilted beams leading displacements at the $\mathrm{y}$ direction, and one light beam without tilt. Note that even this number of tilted beams is not optimal, it is used because is able to illustrate the validity of our proposed technique. Structured illumination of object can be optimized by studying the effect in resolution of increasing the number of tilted beams. In fact, by increasing this number, larger frequency information of the object may be obtained, but the efficiency of the diffractive lenses generated with the LCoS display may be reduced. Thus, an optimal trade-off between these two specifics can be studied to determine the optimal number of tilted beams selected.
By using five beams, the resulting structured illumination leads to 25 different terms for the relation given in Eq. (3), each one with its corresponding constant phase $e^{i\left(\delta_{p}-\delta_{k}\right)}$. However, in the case of $p=k$ (it happens five times for the current $n=5$ case), the constant phase is equal to the unity and we can rewrite Eq. (3) as the summation of two different addends, as shown in Eq. (4). In fact, whereas the first factor of Eq. (4) includes the five terms sharing the constant phase equal to 1 (accounting for the autocorrelation of different parts of the spectra), the second factor of Eq. (4) includes the remainder 20 correlations (accounting for the correlations that cross different parts of the spectra):

$$
\begin{aligned}
\tilde{I}_{t}(u, v)=\sum_{p=1}^{5} \sum_{k=1}^{5} e^{i\left(\delta_{p}-\delta_{k}\right)} C_{p, k}= \\
=\sum_{k=1}^{5} C_{k, k}+\sum_{p \neq k=1}^{5} e^{i\left(\delta_{p}-\delta_{k}\right)} C_{p, k} .
\end{aligned}
$$

In this equation we have 21 unknowns: $C_{p, k}$. By changing the constant phases $\delta_{p}$ we can obtain 
another interferogram (Eq. (4)). By repeating this operation 21 times we will obtain 21 equations. If the constant phases are well selected these equations will be independent. In particular, by using matricidal notation, the interferogram vector $I$ is equal to the product of the unknown vector $C$ with the constant phases matrix $M$.

$$
I=M \cdot C .
$$

At this point, by using pseudo-inverse of the phases matrix $M$, the coefficients of expanded equation are calculated which actually gives the segments of object spectrum:

$$
C=M^{-1} \cdot I
$$

In this work we have used a certain arrangement of constant phases $(0, \pi, \pi / 2)$, being useful to prove the validity of our proposed technique. However, the chosen arrangement of phases is not optimal. Therefore, an optimization process can be applied to achieve a selection of phases minimizing the noise propagation that have place in the inverting process. To this aim, a certain quality indicator, as the Condition Number, can be used.

Finally, the obtained coefficients $C_{p, k}$, are back shifted to their original positions and they are used to fulfill Eq. (3). The shifting process, necessary to retrieve the correct values for the coefficients $C_{p, k}$, is detailed in Ref. [13]. By obtaining accurate values for the $C_{p, k}$ coefficients, the following synthetized spectrum is obtained:

$$
\tilde{I}_{t}(u, v)=\tilde{O}(u, v) H^{\prime(u, v)} .
$$

Equation (7) gives the synthesized spectrum, having higher spatial frequencies than the limited object spectrum. The inverse Fourier transform of Eq. (7) gives the super-resolved image:

$$
I(x, y)=\left|O(x, y) \otimes h^{\prime}(x, y)\right|^{2} .
$$

In Eq. (8) the PSF of the system is narrower than the PSF in Eq. (1), which is the proof of the enhancement of resolution.

\section{Simulation results}

The proposed technique is tested using 1951 US resolution chart as input object by computer simulation. The input object is simultaneously illuminated by five shifted illumination (two along $x$-axis two along $y$-axis and one at center) to record images at the CCD plane. The shift of each illumination from the central one is related to the dimension of the aperture placed at imaging system (see Fig. 1). All images are overlapped at the CCD plane, to recover the segments of object spectrum independently, different phase relations are used.

Figure 2 shows the simulated result in which different recovered parts of the object spectrum are shown. Figures 2(a) and 2(b) show the recovered part of the object spectrum along $x$ axis and Figs. 2(c) and 2(d) show the recovered part of the object spectrum along $y$-axis. The detail of reconstruction is explained in mathematical part of the article. Figure 3(a) shows the reconstructed spectrum and its corresponding image in Fig. 3(b). The reconstructed spectrum is obtained by stitching all the segments of the object spectrum passed through aperture due to different illumination. The inverse Fourier transform of the reconstructed spectrum gives image which is super resolved.

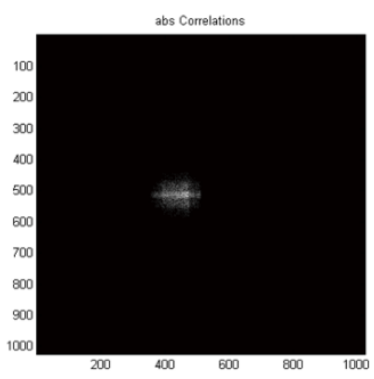

(a)

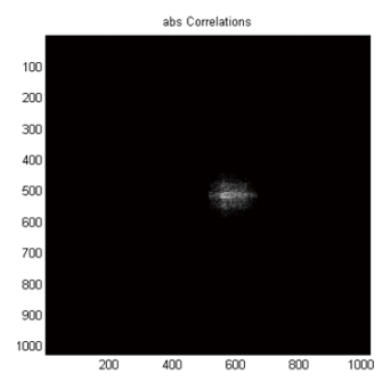

(b)

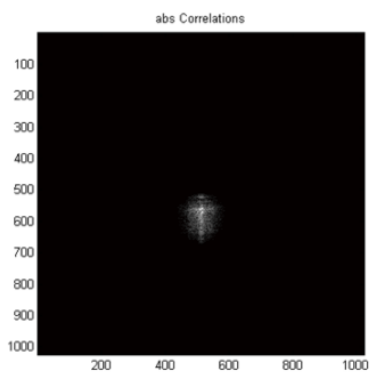

(c)

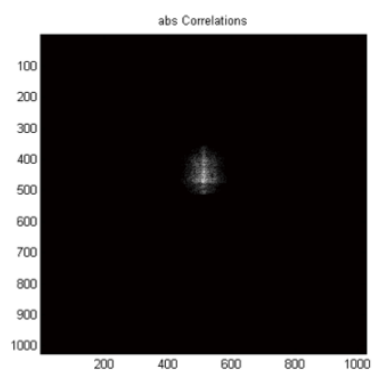

(d)

Fig. 2. Different spectra reconstruction: (a-d) are obtained after solving the linear equation system composed of 21 correlation terms. 


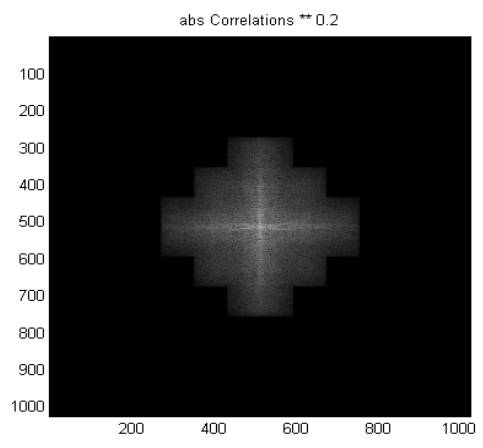

(a)

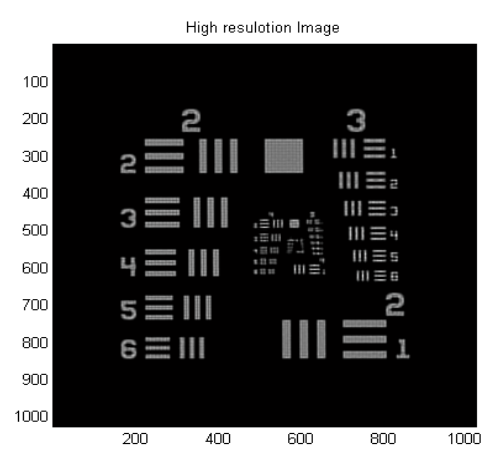

(b)

Fig. 3. Reconstructed spectrum and its corresponding image: (a) show the reconstructed spectrum obtained by combining all the segments of the object spectrum; (b) shows the super resolved image obtained by taking inverse Fourier transform of Fig. 3(a).

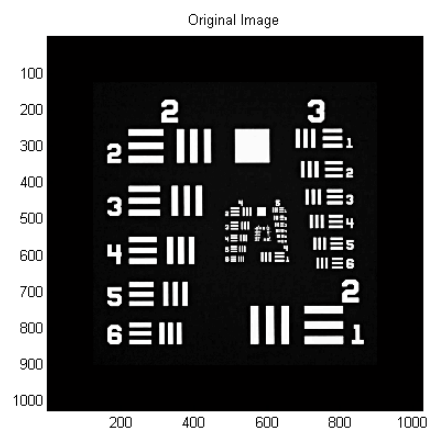

(a)

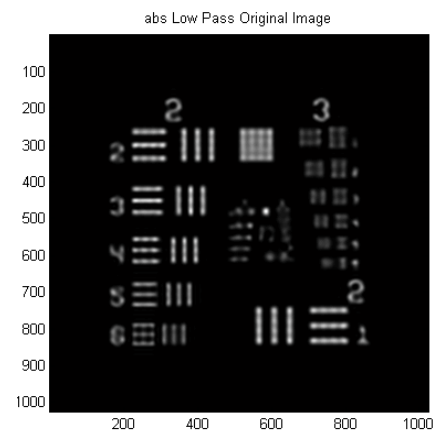

(b)

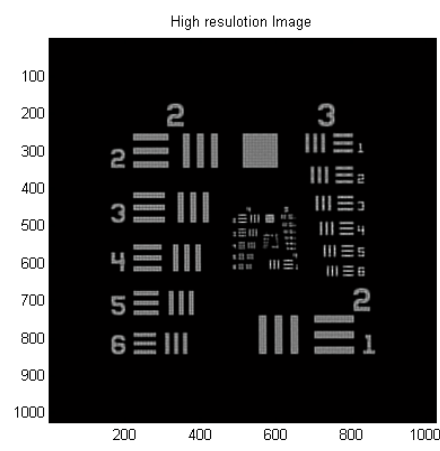

(c)

Fig. 4. Simulations results :(a) Original image; (b) low resolution image obtained with the system; (c) higher resolution image obtained by applying the proposed method.

In addition, we want to emphasize that the results provided in Fig. 4 constitute a generalization of the study given in Ref. [13], where the simultaneous illumination based technique was applied to the one-dimensional case. In this section, the super-resolved method is extended to the two dimensional case, leading to significant improvement in the image resolution. This fact can be observed in Fig. 4(c), where an increase in resolution is obtained not only for the $x$ direction (vertical lines) but also for the $y$ direction (horizontal lines).

Finally, Fig. 4 shows the comparison of band limited image with super resolved image along with original object. Figure $4(\mathrm{~b})$ is the band limited image which is obtained with only one illumination, while Fig. 4(c) shows the super resolved image which is obtained by tilted beam illuminations. Figure 4(a) shows the original object which is used as input object in simulation. The visual comparison clearly shows that Fig. 4(c) is highly resolved compared to Fig. 4(b) which means that the missing frequencies are achieved in Fig. 4(c) using tilted illumination. In Fig. 4(c) the group of three lines under the digit ' 3 ' is very much clearer than the original object. In Fig. 4(b) the same part of the object is blurred, being difficult to identify the number of lines. This is because only low spatial frequencies are passed through aperture while blocked higher spatial frequencies.

\section{Conclusion}

We have presented some additional results for a recently proposed method [13] to achieve superresolution in an optical imaging system. In particular, we have used a $4 f$ optical system to better show the behavior of the method. The proposed method consists in the simultaneous 
illumination of the object with different tilted beams. Each tilted beam allows a different part of the object to pass through the aperture of the system. To obtain the amplitude and phase, an in line interferometric method is used. All tilted illuminations are used simultaneously. By adding different constant phases to each tilted beam, it is possible to extract each part of the spectrum but shifted from its position. Then, the final step is to shift and combine all the spectrums to obtain the superresolved image. We have developed some simulations and the obtained results confirm the proposed method.

\section{Acknowledgements}

We acknowledge financial support from Spanish Ministerio de Economía y Competitividad (FIS2012-39158-C02-01). Anwar Hussain acknowledges Higher Education Commission (HEC) of Pakistan for funding the grant. 